\title{
Obtención y Evaluación de la Estabilidad de Antocianinas de Berenjena (Solanum melongena L.) en Bebidas
}

\author{
Guillermo Arrazola, Irina Herazo y Armando Alvis \\ Universidad de Córdoba. Programa de Ingeniería de Alimentos. Facultad de Ingenierías. Grupo de \\ Investigación Procesos y Agroindustria de vegetales. Carrera 6 № 76-103, Km 3, vía Cereté, Córdoba- \\ Colombia. (e-mail: guillermo.arrazola@ua.es)
}

Recibido Oct. 15, 2013; Aceptado Dic. 11, 2013; Versión final recibida Dic. 18, 2013

\begin{abstract}
Resumen
Se obtuvo extractos de antocianinas de berenjena (Solanum melongena L.) con maltodextrina mediante secado por aspersión, evaluándose la estabilidad del pigmento en polvo en bebidas isotónicas y bebidas a base de aloe vera. Las condiciones de secado por aspersión fueron temperatura de entrada de aire de $180^{\circ} \mathrm{C}$ y concentración de sólidos en la alimentación del 30 \% (en masa). En las bebidas con pigmento se analizaron los cambios en color y contenido de antocianinas durante el almacenamiento del producto a $4{ }^{\circ} \mathrm{C}$ y $25^{\circ} \mathrm{C}$. La temperatura de almacenamiento influyó en la estabilidad de antocianinas y también en los parámetros de color, siendo la temperatura de $25^{\circ} \mathrm{C}$ la que produce mayor velocidad de degradación en ambas bebidas. La bebida isotónica y la bebida a base de aloe vera con maltodextrina y almacenada a $4{ }^{\circ} \mathrm{C}$ presentaron mayor retención de antocianinas (54 y $77.5 \%$ respectivamente).
\end{abstract}

Palabras clave: berenjena, antocianinas, encapsulación, estabilidad, bebida

\section{Obtaining and Evaluation of Stability of Eggplant Anthocyanins (Solanum melongena L.) in Beverages}

\begin{abstract}
Anthocyanins extracts of eggplant (Solanum melongena L.) with maltodextrin by spray drying were obtained, evaluating the stability of the pigment powder in isotonic drinks and aloe vera based drinks. The spray drying conditions were inlet air temperature of $180^{\circ} \mathrm{C}$ and concentration of solids in the feed of $30 \%$ (by mass). In the pigmented beverages changes in color and anthocyanin content of the product during storage at $4{ }^{\circ} \mathrm{C}$ and $25^{\circ}$ $\mathrm{C}$ were analyzed. Storage temperature had influence on anthocyanin stability and also on the color parameters. The highest degradation kinetics in both beverages occurred at $25{ }^{\circ} \mathrm{C}$. Isotonic drinks and aloe vera extracts with the addition of maltodextrin and stored at $4^{\circ} \mathrm{C}$ exhibited higher retention of anthocyanin (54 and $77.5 \%$ respectively).
\end{abstract}

Keywords: eggplant, anthocyanin, encapsulation, stability, beverages 


\section{INTRODUCCIÓN}

Las antocianinas son compuestos fenólicos flavonoides que poseen algunos efectos terapéuticos positivos, principalmente asociados con su capacidad antioxidante. Son un grupo de pigmentos hidrosolubles de origen natural que imparten la coloración roja, púrpura y azul a muchos vegetales y frutos como cerezas, ciruelas, fresas, frambuesas y moras, entre otras (Fennema, 2000; Castañeda et al., 2009). La berenjena morada también pertenece a los frutos que contienen pigmentos antocianos en su cascara a (Noda et al., 2000; Wu y Prior, 2005; Ichiyanagi et al., 2006; Calogero y Di Marco, 2008; Todaro et al., 2009).

En los últimos años, las tendencias mundiales indican un interés acentuado de los consumidores por reemplazar el uso de colorantes artificiales por colorantes de origen natural, que además de ser atractivos para los consumidores aporten beneficios a las funciones biológicas del organismo humano y sean estables en el tiempo (Escalona, 2004; Ribeiro y Stringheta, 2006). Sin embargo la mayoría de los pigmentos de origen natural tienen como limitante su baja estabilidad al sufrir diversas transformaciones en el tiempo (Ribeiro y Stringheta, 2006), razón por la que muchas investigaciones se orientan no solo a la obtención de pigmentos naturales sino también a la búsqueda de alternativas de solución que aumenten su estabilidad.

La propiedad de las antocianinas de ser solubles en agua facilita su incorporación en numerosos sistemas acuosos alimenticios, cualidad que hace que las antocianinas sean colorantes naturales atractivos (Longo y Vasapollo, 2006). Sin embargo, las antocianinas aisladas son altamente inestables y muy susceptibles a la degradación durante el almacenamiento y el procesamiento. Su estabilidad se ve afectada por varios factores tales como $\mathrm{pH}$, temperatura de almacenamiento, estructura química, concentración, luz, oxígeno, solventes, presencia de enzimas, flavonoides, proteínas e iones metálicos; de esta forma su inestabilidad es una limitante para su aplicación como colorante comercial en la industria de alimentos (Owusu, 2005; Castañeda et al., 2009; Olaya et al., 2009).

La microencapsulación es definida como una tecnología de empaque de materiales sólidos, líquidos o gaseosos en miniatura, que consiste en la incorporación de ingredientes alimentarios, enzimas, células u otros materiales en pequeñas cápsulas (Desai y Park, 2005). Implica el recubrimiento de un ingrediente sensible, ya sea puro o una mezcla, dentro de un material para otorgar protección contra la humedad, calor u otras condiciones extremas, para mejorar su estabilidad y aumentar su vida útil (Villena et al., 2009). De esta manera la microencapsulación es una técnica, cuya finalidad en la industria de alimentos es aumentar la vida útil de los ingredientes encapsulados, protegiéndolos contra oxidación química o de los factores ambientales, como es el caso de las vitaminas, pigmentos y otros compuestos bioactivos (Ribeiro y Stringheta, 2006), en comparación con las bebidas usando extracto no microencapsulado."

El objetivo de esta investigación fue obtener extractos de antocianinas de berenjena con maltodextrina mediante secado por aspersión y evaluar la estabilidad del pigmento en polvo en bebidas isotónica y a base de aloe vera.

\section{MATERIALES Y MÉTODOS}

\section{Obtención de antocianinas a partir de cáscara de berenjena}

Se realizó la recolección de berenjenas (Solanum melongena L.) morada negra cultivada en el departamento de Córdoba, caserío retiro de los indios. La recolección de las berenjenas se realizó en la fase de maduración comercial. Para la extracción de antocianinas de la cáscara de berenjena, las muestras fueron peladas, empleando un cuchillo de acero inoxidable. Se extrajo capas de cáscara de berenjena con un espesor de $1 \mathrm{~mm}$, y fueron acondicionadas para obtener un tamaño de partícula de $2 \mathrm{~mm}$ de ancho por $2 \mathrm{~mm}$ de largo aproximadamente (Todaro et al., 2009). Se realizó la extracción del pigmento empleando como solvente etanol acidificado al $50 \%$ durante tres horas de extracción a $30^{\circ} \mathrm{C}$.

\section{Microscopia electrónica de Barrido (SEM) de polvos secos en Spray}

La morfología de las partículas se evaluó por microscopía electrónica de barrido (SEM), en la universidad de Antioquia. Los polvos fueron unidos a una cinta adhesiva de doble cara montado sobre talones de SEM, recubierto con 3-5 mA oro / paladio al vacío y se examinaron con un microscopio electrónico de barrido JEOL® modelo JSM 6490 LV, operado en el modo de alto vacío. 


\section{Evaluación de la estabilidad de antocianinas en bebida isotónica y bebida aloe vera}

Como matriz alimenticia se empleó una bebida isotónica y una a base de aloe vera. La bebida isotónica se preparó en función de la composición y el pH de bebidas comerciales descritas por (Obón et al., 2009; Burin et al., 2011). La bebida Aloe vera fue suministrada por la empresa Aloe Technology. Las antocianinas encapsuladas por aspersión y sin microencapsular (Control) se incorporaron a las bebidas, la mezcla se sometió a agitación hasta la disolución del pigmento microencapsulado. Las muestras fueron almacenadas en frascos estériles protegidos de la luz a temperaturas de $4{ }^{\circ} \mathrm{C}$ y temperatura ambiente $25^{\circ} \mathrm{C}$.

Se evaluó la vida de anaquel de las antocianinas encapsuladas en la bebida en relación a los cambios de color, contenido de antocianinas, y actividad antioxidante durante el almacenamiento del producto a temperaturas de refrigeración $\left(4^{\circ} \mathrm{C}\right)$ y ambiente $\left(25^{\circ} \mathrm{C}\right)$. Los análisis se realizaron cada 5 días durante 40 días de almacenamiento. Para evaluar la estabilidad de las antocianinas extraídas de berenjena en jugos, se desarrolló un diseño experimental completamente aleatorizado, unifactorial. En donde el tiempo se analizó a 8 niveles. Se aplicó análisis de regresión lineal para determinar el modelo que describe la cinética de degradación de antocianinas y de color.

\section{Determinación Contenido de antocianinas en bebidas}

Se realizó la determinación del total de monómeros de antocianinas por el método diferencial de pH descrito por Giusti y Wrolstad (2001) y Poo (2005). El extracto se diluyó con dos buffer (pH 1,0 y pH 4,5). Para poder medir la absorbancia. El factor de dilución fue el mismo para ambas muestras. A las muestras diluidas se les realizó la medición de absorbancia a la longitud de onda de máxima absorción (520nm) (Giusti y Wrolstad 2001; Poo 2005). El contenido de pigmentos en el extracto se expresó en $\mathrm{mg} / 100 \mathrm{~g}$. $R^{2}$ de 0.99 , ecuación de la curva, $Y=$ $m a+b, a=1,70917$ y $b=-2,10308$. (Antocianinas)

Análisis de color en bebidas.

Los parámetros de color $L^{*}, a{ }^{*}, b^{*} C^{*}$ (Saturación de color) y ${ }^{\circ} \mathrm{H}$ (Angulo de tono) se midieron con un colorímetro Colorflex EZ 45 (HunterLab®). El colorímetro se calibró con un plato de cerámica estándar de color verde y blanco estándar antes de su lectura.

\section{Determinación de poder antioxidante en bebidas}

La actividad antioxidante de los polvos se midió empleando el método ABTS* (6-sulfonato-3-etilbenzotiazolina), las mediciones de absorbancia se realizaron a $732 \mathrm{~nm}$ empleando un espectofometro (Genesis 20). Para la evaluación se emplearon $10 \mu \mathrm{L}$ del extracto diluido en DMSO (Dimetilsufoxido) y $990 \mu \mathrm{L}$ de la solución del radical $\mathrm{ABTS}^{*}$. Luego de 30 min de reacción a temperatura ambiente y en la oscuridad, se leyó el cambio en la absorbancia de la muestra. Los resultados se expresaron como IC50, el cual corresponde a la concentración necesaria para inhibir el $50 \%$ de la absorbancia del radical. También se calcularon valores TEAC mediante la construcción de una curva patrón usando diferentes concentraciones de TROLOX® (ácido 6-hidroxi-2,5,7,8tetrametil-2-cromanocarboxílico) y Ácido Ascórbico (Alvis et al., 2012). $\mathrm{R}^{2}$ de 0.99 ecuación de la curva, $\mathrm{Y}=\mathrm{m} \mathrm{a}+\mathrm{b}, \mathrm{a}=19.098$ y $\mathrm{b}=9.775$. (Capacidad antioxidante)

\section{Análisis estadístico de los datos}

En la evaluación de la estabilidad de los extractos de antocianinas de berenjena en bebidas, se realizaron análisis de varianzas, test de diferencias de medias (Tukey) y ajuste de regresiones con significancia del $5 \%$. Estos análisis se realizaron en el programa SAS versión 9.2.

\section{RESULTADOS Y DISCUSIÓN}

\section{Propiedades de antocianinas microencapsuladas}

La morfología de las microcápsulas a concentraciones de $30 \%$ de sólidos en la alimentación y con temperatura de salida de aire a $180^{\circ} \mathrm{C}$, son de tamaño y forma variable, es decir hay capsulas de forma esférica con una superficie lisa y capsulas con superficie abollada o irregular, como se muestra en la figura 1. Esta estructura lisa y esférica de microencapsulados con maltodextrina ha sido observada por autores como Tonon et al. (2008); Escalona (2004); Caparino et al. (2012); Ersus y Yurdagel (2007) en sus estudios de microencapsulación por 
secado aspersión empleando maltodextrinas en extractos de Acai, luteina-enocianina, mango y pigmentos zanahoria negra (Daucus carota L) respectivamente. Asimismo Olaya et al. (2009) reportaron partículas que exhibían superficie lisa, y otras que presentaban superficies porosas con abolladuras superficiales en la encapsulación con maltodextrina de pigmentos de antocianos de Mora de Castilla (Rubus glaucus) y tamarillo (Solanum betaceum).


Fig. 1. Fotografías de Microscopía Electrónica de Barrido (SEM) de microcápsulas a) 30 \% MD y $180^{\circ} \mathrm{C}, 1000 x$. b) $30 \% \mathrm{MD}$ y $180^{\circ} \mathrm{C}, 5000 x$.

\section{Estabilidad de contenido de antocianinas en bebidas}

El pigmento de antocianinas de cáscara de berenjena microencpasulado mediante la técnica de secado por aspersión se incorporó fácilmente y en forma homogénea en la bebida isotónica y en la bebida a base de sábila. La figura 2 muestra el contenido de antocianinas en el tiempo para las dos bebidas en estudio. En las dos bebidas, la degradación de antocianinas de cáscara de berenjena exhibió una cinética de primer orden (figura 2). Los valores de $R^{2}$ estuvieron en un rango de 0.89 y 0.98 lo que indica un buen ajuste de los datos al modelo cinético de primer orden.
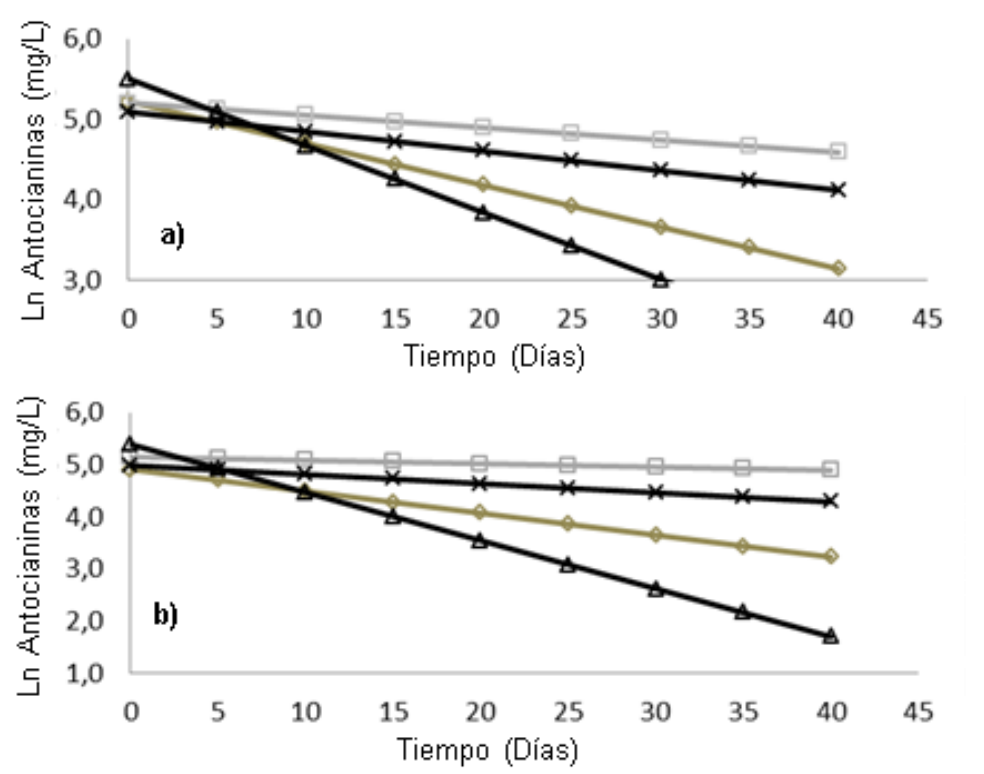

Fig. 2. Contenido de antocianinas en: a) bebida Isotónica y b) bebida Aloevera durante el tiempo de

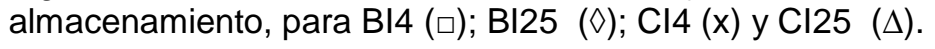

Los parámetros del modelo se muestran en la Tabla 1. El contenido de antocianinas en todas las bebidas en estudio disminuyó a medida que el periodo de almacenamiento se incrementó. Este comportamiento también ha sido reportado por otros autores, tales como (Wang y Xu, 2007; De Rosso y Mercadante, 2007; Tonon et al., 2010; Burin, et al., 2011). A través del análisis de la varianza (ANOVA) realizado sobre los valores de vida 
media de las bebidas, es posible verificar que el factor temperatura y tratamiento influyó significativamente $(p<$ $0,05)$, en el tiempo de vida media y porcentaje de retención de antocianinas. De esta manera la temperatura de almacenamiento $4 \pm 1^{\circ} \mathrm{C}$ exhibieron mayores valores de vida media del pigmento que las muestras a $25 \stackrel{\circ}{\circ}$ (Tabla 1). Asimismo la temperatura de almacenamiento a $25{ }^{\circ} \mathrm{C}$ mostró muy bajos valores de retención de antocianinas. Esta influencia negativa de la temperatura en la estabilidad de antocianinas ha sido observada por muchos investigadores (Falcao 2003; Falcao et al. 2008). Tonon et al. (2010) observaron que el aumento de la temperatura conduce a una degradación más rápida de antocianina, debido a que los pigmentos antocianos son muy termo sensible. El mecanismo de degradación de antocianinas por calor ocurre probablemente debido a la apertura del anillo de catión flavylium, seguido por conversión a la forma chalcona, que es incolora y es una degradación irreversible (Falcao 2003; Malacrida y Da Motta 2006).

Tabla 1. Parámetros cinéticos de degradación de antocianinas en bebidas almacenadas a 4 y $25^{\circ} \mathrm{C}$.

\begin{tabular}{|c|c|c|c|c|}
\hline \multirow[t]{2}{*}{ Tratamiento } & \multicolumn{3}{|c|}{ Antocianinas (mg/L) } & \multirow[b]{2}{*}{$\% R$} \\
\hline & $\mathrm{K}(\mathrm{h})$ & $t_{1 / 2}$ & $R^{2}$ & \\
\hline $\mathrm{BI} 4^{*}$ & -0.0064 & - & 0.89 & 54.0 \\
\hline $\mathrm{BI} 25$ & -0.0021 & 319.9 & 0.98 & 12.5 \\
\hline $\mathrm{Cl} 4$ & -0.0010 & 684.3 & 0.98 & 37.7 \\
\hline $\mathrm{Cl} 25$ & -0.0035 & 200.2 & 0.92 & 3.60 \\
\hline BA4 & -0.00026 & - & 0.92 & 77.5 \\
\hline BA25 & -0.0017 & 395.9 & 0.91 & 18.6 \\
\hline CA4 & -0.00073 & 957 & 0.94 & 49.7 \\
\hline CA25 & -0.0038 & 180.5 & 0.92 & 2.50 \\
\hline
\end{tabular}

La bebida isotónica y Aloe vera adicionada del extracto microencapsulado con maltodextrina y almacenada a 4 ${ }^{\circ} \mathrm{C}$, fueron las que presentaron los mayores porcentajes de retención (\% R) del compuesto después de 960 horas de estudio, los \% R para estas bebidas fueron 54 y $77.5 \%$ respectivamente. Un comportamiento similar observaron Falcao et al. (2008) en extracto de antocianinas de uva adicionados a una bebida hidratada, en donde a $4{ }^{\circ} \mathrm{C}$ obtuvo \% de retención de 76 y $79 \%$ a 217 horas de evaluación. Este autor obtuvo valores de vida media en su bebida a $4^{\circ} \mathrm{C}$ de 662 y 533 horas, los cuales son valores muchos más bajos que los obtenidos en esta investigación. Kirca y Cemeroglu (2003) han observado degradación de las antocianinas en jugo de naranja Roja $\left(69^{\circ}\right.$ brix), con tiempos de vida media de 116 y 17.7 horas a temperatura de $5{ }^{\circ} \mathrm{C}$ y de $25^{\circ} \mathrm{C}$ respectivamente. Otros autores también han obtenido similares resultados al evaluar el contenido de antocianinas en el tiempo a diferentes temperaturas de almacenamiento. Wang y Xu (2007) reportaron t $1 / 2$ de 330 y 32 días para jugos de mora ( $8.9^{\circ}$ brix) a 5 y $25^{\circ} \mathrm{C}$ respectivamente. Los resultados estadísticos también muestran que hay una interacción significativa entre las variables tratamientos y temperatura $(p<0,05)$ tanto para la bebida Isotónica como para la de aloe vera. Las muestras de bebidas isotónicas mantenidas a $4{ }^{\circ} \mathrm{C}$ con adición de antocianina microencapsulada no alcanzaron a degradarse en un $50 \%$ durante su tiempo de estudio, mientras que la bebida isotónica adicionada de extracto sin microencapsular mostró un $\mathrm{t}_{1 / 2} 684$ horas y $\%$ R de $37.7 \%$.

Ribeiro y Stringheta (2006) analizaron el comportamiento de polvos microencapsulados con maltodextrina adicionados a soluciones buffer de $\mathrm{pH}$ de 2, 3 y 4 y protegidos de la luz, obteniendo buenos resultados de $\mathrm{t}_{1 / 2}$ con valores de 141, 116 y 86 días. Los valores de $t_{1 / 2}$ fueron mayores que el los del control (extracto liquido) con $\mathrm{t}_{1 / 2}$ de 135, 105 y 75 días. De Rosso y Mercadante (2007) realizaron la adición de extracto concentrado de Acerola (Malpighia emarginata ) y Acai (Euterpe oleracea Mart) en bebida isotónica almacenada a $20^{\circ} \mathrm{C}$, y encontraron $t_{1 / 2}$ de 11.4 horas y 943 horas respectivamente. Bordignon-Luiz et al. (2007) estudiaron la adición de antocianinas en bebida rehidratada y encontraron valores de $t_{1 / 2}$ de 330 a 737 horas a temperaturas de almacenamiento de 4 y $24{ }^{\circ} \mathrm{C}$ respectivamente. Con porcentajes de retención de 38 y $75 \%$ evaluadas por 45 días. Burin et al. (2011) observaron que polvos microencapsulados de extractos de uva con maltodextrina, adicionado a bebida isotónica, presentaron un $\mathrm{t}_{1 / 2}$ de 556 horas y \% $\mathrm{R}$ de 35.9 a $25 \stackrel{\circ}{\circ} \mathrm{C}$; sin embargo los tratamientos mantenidos a $4{ }^{\circ} \mathrm{C}$ no habían alcanzado el $\mathrm{t}_{1 / 2}$ después de 960 horas de estudio, el cual presentó valores de \% R de $94 \%$, los cuales son valores mayores a los obtenidos en el presente estudio. Martínez et al. (2011), encontraron que el almacenamiento a $37{ }^{\circ} \mathrm{C}$ resultó en una mayor degradación de las antocianinas respecto a la temperatura de refrigeración $\left(4^{\circ} \mathrm{C}\right)$; así, el tiempo de vida media $t_{1 / 2}$ para las antocianinas a 19,5 ${ }^{\circ}$ Brix fue de 7,5 semanas a $4^{\circ} \mathrm{C}$ y sólo de 3,0 semanas a $37^{\circ} \mathrm{C}$. 


\section{Estabilidad de Parámetros de Color en Bebidas}

Los parámetros $\mathrm{L}^{*} \mathrm{C}^{*} \mathrm{y}{ }^{\circ} \mathrm{H}$ presentaron cambios notables en las bebida de aloevera e isotónicas almacenadas a diferentes temperaturas durante los 40 días de evaluación, esto confirma la degradación de los atributos de color visuales en las bebidas. En este estudio, los parámetros de color $\left(L^{*}, C^{*} y{ }^{\circ} H\right)$ fueron analizados a través de regresión lineal con respecto al tiempo (figura 3), los parámetros cinéticos se reportan en la tabla 2.

Tabla 2. Parámetros cinéticos de degradación del color en bebidas

\begin{tabular}{|c|c|c|c|c|c|c|c|c|c|}
\hline \multirow[t]{2}{*}{ Tratamiento } & \multicolumn{3}{|c|}{$\mathrm{C}^{*}$} & \multicolumn{3}{|c|}{${ }^{\circ} \mathrm{H}$} & \multicolumn{2}{|l|}{$\mathrm{L}^{*}$} & \multirow[b]{2}{*}{$\mathrm{R}^{2}$} \\
\hline & $\mathrm{K}(\mathrm{h})$ & $t_{1 / 2}$ & $R^{2}$ & $\mathrm{~K}(\mathrm{~h})$ & $t_{1 / 2}$ & $R^{2}$ & $\mathrm{~K}(\mathrm{~h})$ & $t_{1 / 2}$ & \\
\hline $\mathrm{BI} 4$ & -0.0011 & 604 & 0.96 & 0.0037 & 188 & 0.92 & 0.0021 & 251 & 0.95 \\
\hline $\mathrm{BI} 25$ & -0.0019 & 359 & 0.95 & 0.066 & 10.5 & 0.98 & 0.0022 & 314 & 0.94 \\
\hline $\mathrm{Cl} 4$ & -0.0010 & 674 & 0.94 & -0.015 & 45.2 & 0.95 & 0.0009 & 796 & 0.92 \\
\hline $\mathrm{Cl} 25$ & -0.0018 & 377 & 0.96 & -0.019 & 35 & 0.94 & 0.0006 & - & 0.95 \\
\hline BA4 & -0.0014 & 487 & 0.94 & -0.031 & 22 & 0.91 & 0.0015 & 456 & 0.96 \\
\hline BA25 & -0.0037 & 188 & 0.98 & -0.055 & 12.6 & 0.94 & 0.0026 & 268 & 0.96 \\
\hline CA4 & -0.0014 & 499 & 0.91 & -0.038 & 18.2 & 0.93 & 0.0008 & - & 0.90 \\
\hline CA25 & -0.0028 & 249 & 0.97 & -0.074 & 9.3 & 0.97 & 0.0019 & 371 & 0.94 \\
\hline
\end{tabular}

$\mathrm{BI}^{*}$ y BI25: Bebida isotónica con microcápsula a 4 y 25ํㅡ respectivamente; $\mathrm{Cl} 4$ y Cl25: Bebida isotónica con extracto sin microencapsular a 4 y 25우 BA4 y BA25: Bebida Aloe vera con Microcápsula a 4 y 25두 CA4 y CA25: Bebida Aloevera con extracto sin microencapsular a 4 y 25으. Esta notación se empleará en el resto del documento.

Los valores de $L^{*}$ para todos los tratamientos durante el tiempo estudiado, aumentaron en el tiempo. Sin embargo los valores iniciales y finales de $L$ en los tratamientos fueron bajos, es decir, las muestras presentaron $L^{*}$ entre 2 y 6.5; esto indica que todos los tratamientos se mantuvieron en el mismo tono de color oscuro durante todo el tiempo de almacenamiento (figura $3 a$ y 3b). Duangmal et al. (2008) observaron un comportamiento similar, en donde $L^{*}$ no cambió durante el tiempo de almacenamiento de microencapsulados de Flor de Jamaica con maltodextrina y trehalosa. Obon et al. (2009) también observaron un aumento en el valor $L$ en bebidas isotónicas almacenadas a $4{ }^{\circ} \mathrm{C}$.

El ángulo ${ }^{\circ} \mathrm{H}$ en la bebida isotónica a $25^{\circ} \mathrm{C}$, fue significativamente diferente durante los tiempos estudiados. De esta manera el $\mathrm{H}^{\circ}$ varió de 2.5 a $57.6{ }^{\circ} \mathrm{C}$, es decir que la BI25 pasó desde el color rojo hasta el color anaranjado. El tratamiento BI4 no presentó cambio en el color rojo, oscilando el valor ${ }^{\circ} \mathrm{H}$ de 2.5 a 5.2 . En la Tabla 2 se puede observar los valores de la constante $\mathrm{K}$, el cual muestra que hay diferencia en la velocidad de degradación para la bebida isotónica adicionada de extracto microencapsulado a diferentes temperaturas. En las bebidas $\mathrm{Cl} 25$ y Cl 4 el valor de $\mathrm{H}$ mostró un cambio del color rojo al color morado o violeta, donde el ${ }^{\circ} \mathrm{H}$ varió desde -3.4 a -20.3 y -3.4 a -17.3 durante su almacenamiento a 25 y $4{ }^{\circ} \mathrm{C}$ respectivamente (figura $3 \mathrm{c}$ y $3 \mathrm{~d}$ ).

Los valores iniciales de las bebidas isotónicas muestran que el color de todos los sistemas adicionadas con extracto microencapsulado fue un color rojo mientras que la observada en los sistemas con adición de extracto sin microencapsular la tonalidad del color fue hacia el morado o violeta. De Rosso y Mercadante (2007) observaron en bebida isotónica adicionada de extractos de Acai (Euterpe oleracea Mart.) y Acerola (Malpighia emarginata), almacenada a $20^{\circ} \mathrm{C}$, un cambio del color rojo a amarillo, ya que los valores de ${ }^{\circ} \mathrm{H}$ aumentaron durante el tiempo del experimento.

Para la bebida a base de aloe vera el ángulo ${ }^{\circ} \mathrm{H}$ para BA25 y BA4 fue diferente significativamente durante los tiempos estudiados. Aunque ambas bebidas tuvieron la tendencia de cambio del color rojo al violeta o morado, fue la BA25 la que presentó la mayor velocidad de degradación o cambio del color (Tabla 2). Para las bebidas a base de aloe vera adicionadas de extracto no microencapsulado, el $\mathrm{H}^{\circ}$ presentó diferencia significativa en CA25, disminuyendo significativamente en el tiempo cero desde $-6.7\left(353.2^{\circ}\right)$ hasta las 860 horas con un ${ }^{\circ} \mathrm{H}$ de $-75.1\left(285^{\circ}\right)$. Estos resultados muestran que la bebida presentó un cambio del color rojo, pasando por el violeta y el color azul durante su almacenamiento. La CA4 tuvo una disminución del ${ }^{\circ} \mathrm{H}$ de $6.7\left(353.2^{\circ}\right)$ a $45.53(314.5$ $\left.{ }^{\circ}\right)$, presentando cambio de color del rojo al violeta. Los cambios en el valor ${ }^{\circ} \mathrm{H}$ podrían estar asociados a la degradación de antocianinas y a la posible formación de especies chalconas (Reyes y Cisneros, 2007). 

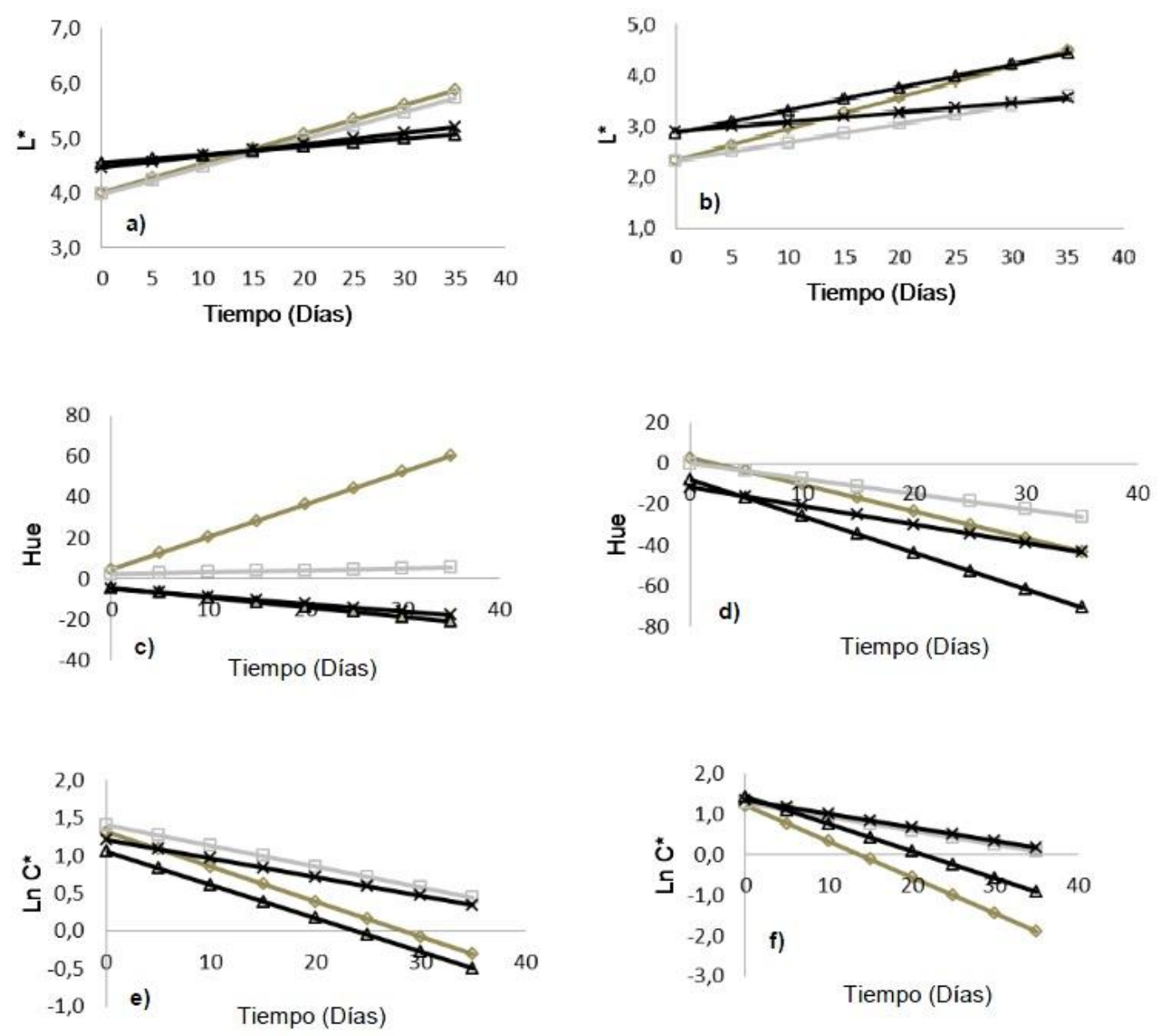

Fig. 3. Parámetro de color en bebidas durante el almacenamiento, a) $L^{*}$ Bebida isotónica, b) $L^{*}$ Bebida Aloe vera, c) Hue Bebida isotónica, d) Hue Bebida Aloe vera, e) Ln C* Bebida Isotónica) f) Ln C* bebida Aloe vera

La bebida isotónica y de Aloe vera adicionada con extracto microencapsulado y sin microencapsular, almacenadas a diferentes temperaturas, presentaron valores bajos de tonalidad o valor $\mathrm{C}^{*}$, el cual disminuyó al transcurrir el tiempo de almacenamiento (figura 3 e y $3 \mathrm{f}$ ). Para todas las bebidas empleadas en este estudio el $t_{1 / 2}$ de $C^{*}$ fue más bajo al aumentar la temperatura de almacenamiento, de esta manera el color de las muestras fue gradualmente tornándose más opaco. Varios autores han observado este comportamiento del tiempo y la temperatura en el parámetro de color $C^{*}$ (Reyes y Cisneros, 2007; De Rosso y Mercadante, 2007; Duangmal et al., 2008; Obón, et al., 2009). Los cambios en C* están fuertemente correlacionados a los cambios en la cantidad de antocianinas y por lo tanto se consideraron un buen indicador del contenido de antocianinas (Reyes y Cisneros, 2007; Duangmal et al., 2008). La temperatura de almacenamiento de las muestras afectó significativamente los parámetros de color. Este mismo comportamiento fue observado por Sari et al. (2012) en bebidas adicionadas de extractos de antocianinas de Jambolan (Syzygium cumini). Por otro lado en cuanto la diferencia general de color $(\Delta \mathrm{C})$, los mayores valores de este parámetro se obtuvo con las bebidas isotónicas y aloe vera a $25^{\circ} \mathrm{C}$ adicionadas de microcápsulas secas en spray, con valores de 4.58 y 4.81 respectivamente. Sin embargo a temperatura de almacenamiento de $4{ }^{\circ} \mathrm{C}$ se observaron valores de variación de color mucho más bajo, siendo el valor menor el de la bebida isotónica control almacenada a $4{ }^{\circ} \mathrm{C}$, con $\Delta \mathrm{C}$ de 2.2 , seguido por las bebidas isotónica y de aloe vera adicionada con extracto microencapsulado con $\Delta \mathrm{C} 3.5$ y 3.7 respectivamente. Una diferencia de color baja, es deseable debido a que indica que el color de la bebida se mantuvo durante el tiempo de almacenamiento. Obón et al. (2009) reportaron que una diferencia de color general de 0-1.5 puede considerarse pequeña, lo que indica que la muestra es casi idéntica a la original por observación visual. Sin embargo cuando el valor de $\Delta \mathrm{C}$ se encuentra dentro del intervalo de 1.5-5, la diferencia de color ya puede ser distinguida, y esta diferencia se hace evidente cuando el valor de $\Delta \mathrm{C}$ es mayor que 5 (Obón et al., 2009). 


\section{Capacidad antioxidante de bebidas}

En la tabla 3 se pueden observar los valores de actividad antioxidantes, como IC50 para el inicio y final del periodo de almacenamiento de bebidas isotónicas y de aloe vera. El IC50 de bebidas Isotónicas aumentó después de 40 días de almacenamiento de la bebida, lo que indica una disminución de la capacidad antioxidante de la bebida. Sin embargo la capacidad antioxidante en la bebida de Aloe vera y control, no presentó un cambio apreciable incluso para las temperaturas de $25^{\circ} \mathrm{C}$.

Tabla 3. IC50 al inicio y final del almacenamiento de bebidas

\begin{tabular}{|c|cc|}
\hline Tratamiento & IC50 Inicial & IC50* Final \\
\hline BI4 & 131.6 & 577.0 \\
BI25 & 126.7 & 669.7 \\
Cl4 & 191.8 & 907.2 \\
Cl25 & 223.4 & 1165.9 \\
BA4 & 73.9 & 89.0 \\
BA25 & 84.6 & 103.6 \\
CA4 & 71.8 & 117.1 \\
CA25 & 84.4 & 101.2 \\
\hline
\end{tabular}

\section{CONCLUSIONES}

La temperatura de almacenamiento de las bebidas ejerció una influencia significativa en la degradación de las antocianinas, siendo la temperatura ambiente de $25^{\circ} \mathrm{C}$ la que presentó los menores valores de vida media y porcentajes de retención. La bebida isotónica y aloe vera adicionada del extracto microencapsulado con maltodextrina y almacenada a $4{ }^{\circ} \mathrm{C}$, presentaron mayores porcentajes de retención (54 y $77.5 \%$ respectivamente) de antocianinas que las bebidas adicionadas del extracto sin microencapsular. El parámetro de color ${ }^{\circ} \mathrm{H}$ de todas las bebidas almacenadas a $25^{\circ} \mathrm{C}$, adicionadas de extracto microencapsulado y no microencapsulado, presentó un cambio de color del rojo $\left(2.5^{\circ}\right.$ a $\left.-3.4^{\circ}\right)$ hacia las tonalidades moradas $(-17.3$ a 20.3), excepto en la bebida isotónica adicionada de polvo microencapsulado, en donde paso de tonalidad roja $\left(2.5^{\circ}\right)$ a la amarilla $\left(57.6^{\circ}\right.$ ) durante el tiempo de almacenamiento a $25^{\circ} \mathrm{C}$. El IC50 de las bebidas disminuyó con respecto al IC50 al inicio, de esta manera hay evidencia de la degradación de algunos compuestos responsables de esta capacidad antioxidantes, tales como los contenidos de antocianinas.

\section{REFERENCIAS}

Alvis, A., G. Arrazola y W. Martínez, Evaluación de la Actividad y el Potencial Antioxidante de Extractos HidroAlcohólicos de Cúrcuma (Cúrcuma longa). Información Tecnológica. Vol. 23(2), 11-18 (2012).

Bordignon-Luiz, M., C. Gauche, E. Gris y L. Falcao, Colour stability of anthocyanins from Isabel grapes (Vitis labrusca L.) in model systems. LWT 40: 594-599 (2007).

Burin, V., P. Rossa, N. Ferreira-Lima, M. Hillmann y M. Boirdignon-Luiz, Anthocyanins: optimisation of extraction from Cabernet Sauvignon grapes, microcapsulation and stability in soft drink. International Journal of Food Science and Technology 46: 186-193 (2011).

Calogero, G. y G. Di Marco, Red Sicilian orange and purple eggplant fruits as natural sensitizers for dyesensitized solar cells. Solar Energy Materials \& Solar Cells, 92: 1341- 1346 (2008).

Caparino, O., J. Tang, C. Nindo, S. Sablani, J. Powers y J. Fellman, Effect of drying methods on the physical properties and microstructures of mango (Philippine 'Carabao' var.) powder. Journal of Food Engineering 111: 135-148 (2012).

Castañeda, A., M. Pacheco, M. Páez, J. Rodríguez y C. Galán, Chemical studies of anthocyanins: A review. Journal Food Chemistry 113: 859-871 (2009). 
De Rosso, V. y Z. Mercadante, Evaluation of colour and stability of anthocyanins from tropical fruits in an isotonic soft drink system. Innovative Food Science and Emerging Technologies 8: 347-352 (2007).

Desai, K. y H. Park, Recent development in microencapsulation of foods ingredients. Drying Technology, 23 1361:1394 (2005).

Duangmal, K., B. Saicheua y S. Sueeprasan, Colour evaluation of freeze-dried roselle extract as a natural food colorant in a model system of a drink. LWT $41: 1437-1445$ (2008).

Ersus S. y U. Yurdagel, Microencapsulation of anthocyanin pigments of black carrot (Daucuscarota L.) by spray drier. Journal Food Engineering. 80, 805-812 (2007).

Escalona, S. Encapsulados de luteina-enocianina y su aplicación en alimentos. Tesis Ingeniero en Alimentos. Facultad de ciencias químicas y farmacéuticas, universidad de Chile. Santiago de Chile (2004).

Falcão, L., A. Falcão, E. Gris, y M. Bordignon, Spectrophotometric study of the stability of anthocyanins from Cabernet Sauvignon grape skins in a model system. Brazilian Journal of Food Technology 11 (1): $63-69$ (2008).

Falcão, L., Estabilidade de antocianinas extraídas de uvas Cabernet Sauvignon (Vitis vinifera L.) em solução tampão, bebida isotônica e iogurte. Mestre em Ciência dos Alimentos. Universidade Federal de Santa Catarina. Brasil (2003).

Fennema, O., Color y estabilidad de antocianinas. En: Química de los alimentos. Segunda Edición. Editorial Acribia S.A. Zaragoza. P. 807-820 (2000).

Giusti, M. y R. Wrolstad, Characterization and Measurement of Anthocyanins by UV-Visible Spectroscopy. Current Protocols in Food Analytical Chemistry. F1.2.1- F1.2.13 (2001).

Ichiyanagi, T., N. Terahara, M. Rahman y T. Konishi, Gastrointestinal uptake of nasunin, acylated anthocyanin in eggplant. Journal of Agricultural and Food Chemistry, 54:(15): 5306-5312 (2006).

Kirca, A. y B. Cemeroglu, Degradation kinetics of anthocyanins in blood orange juice and concentrate. Food Chemistry 81: 583-587 (2003).

Longo, L. y G. Vasapollo, Extraction and identification of anthocyanins from Smilax aspera L. berries. Food Chemistry 94: 226-231 (2006).

Malacrida, C. y S. Da Motta, Antocianinas em Suco de Uva: Composição e Estabilidade. B.Ceppa, Curitiba 24 (1): 59-82 (2006).

Martínez Z. J., S. H. Rojas, G.G. Borda, C. A. Hastamorir y R. M. Medina, Estabilidad de Antocianinas en Jugo y Concentrado de Agraz (Vaccinium meridionale Sw.). Rev. Fac. Nal. Agr. Medellín 64(1): 6015-6022 (2011).

Noda, Y., T. Kneyuki, K. Igarashi, A. Mori y L. Packer, Antioxidant activity of nasunin, an anthocyanin in eggplant peels. Toxicology 148: 119-123 (2000).

Obón, J., M. Castellar, M. Alacid y J. Fernández, Production of a red-purple food colorant from Opuntia stricta fruits by spray drying and its application in food model systems. Journal of Food Engineering 90:471-479 (2009).

Olaya, C., M. Castaño y G. Garzón, Stability of anthocyanins from Rubus glaucus Benth and Solanum betaceum Cav.dark-red strain as affected by temperature, storage time and water activity. Acta Biológica Colombiana 14 (3): 141-156 (2009).

Owusu, A., Chemistry Postharvest. En: Introduction to Food Chemistry. Primera Edición. Editorial CRC Press. United States of America. P. 219 (2005).

Poo, B., Concentración de Antocianinas en Jugo de Cranberries (Vaccinium macrocarpon Ait.) mediante Nanofiltración. Tesis de Licenciado. Facultad de ciencias agrarias. Universidad Austral. Chile (2005). 
Sari, P., C. Wijaya, D. Sajuthi y U. Supratman, Colour properties, stability, and free radical scavenging activity of jambolan (Syzygium cumini) fruit anthocyanins in a beverage model system: Natural and copigmented anthocyanins. Food Chemistry 132: 1908-1914 (2012).

Reyes, L. F. y L. Cisneros-Zevallos, Degradation kinetics and colour of anthocyanins in aqueous extracts of purple- and red-flesh potatoes (Solanum tuberosum L.). Food Chemistry 100: 885-894 (2007).

Ribeiro, F. y P. Stringheta, Microencapsulamento de antocianinas. Biotecnologia Ciência e Desenvolvimento 36: (2006).

Todaro, A., F. Cimino, P. Rapisarda, A. Catalano, R. Barbagallo y G. Spagna, Recovery of anthocyanins from eggplant peel. Journal Food Chemistry 114, 434-439 (2009).

Tonon, R., C. Brabet y M. Hubinger, Anthocyanin stability and antioxidant activity of spray-dried açai (Euterpe oleracea Mart.) juice produced with different carrier agents. Food Research International, 43 (3), 907-914 (2010).

Tonon, R., C. Brabet y M. Hubinger, Influence of process conditions on the physicochemical properties of acai (Euterpe oleraceae Mart.) powder produced by spray drying. Journal of Food Engineering 88, 411-418 (2008).

Villena, M., H. Morales, L. Gallardo y M. Ruiz, Técnicas de microencapsulación: una propuesta para microencapsular probióticos. Ars Pharm, 50 (1): 43-50 (2009).

Wang, W. y S. Xu, Degradation kinetics of anthocyanins in blackberry juice and concentrate. Journal of Food Engineering 82: 271-275 (2007).

$\mathrm{Wu}, \mathrm{X} . \mathrm{y}$ R. Prior, Identification and characterization of anthocyanins by high-performance liquid chromatography-electrospray ionization-tandem mass spectrometry in common foods in the united states: Vegetables, nuts, and grains. Journal of Agriculture and Food Chemistry, 53: 3101-3113 (2005). 